\title{
Measuring Environmental Quantum Noise Exhibiting a Nonmonotonic Spectral Shape
}

\author{
Y. Romach, $,{ }^{1,}{ }^{*}, \dagger$ A. Lazariev, ${ }^{2, \dagger}$ I. Avrahami, ${ }^{3}$ F. Kleißler, ${ }^{2}$ S. Arroyo-Camejo, ${ }^{2}$ and N. Bar-Gill ${ }^{1,3}$ \\ ${ }^{1}$ The Racah Institute of Physics, The Hebrew University of Jerusalem, 9190401 Jerusalem, Israel \\ ${ }^{2}$ Department of Nanobiophotonics, Max Planck Institute for Biophysical Chemistry, Am Fassberg 11, \\ 37077 Göttingen, Germany \\ ${ }^{3}$ Department of Applied Physics, Rachel and Selim Benin School of Computer Science and Engineering, The \\ Hebrew University of Jerusalem, 9190401 Jerusalem, Israel
}

(Received 22 March 2018; revised manuscript received 3 December 2018; published 31 January 2019)

\begin{abstract}
Understanding the physical origin of noise affecting quantum systems is important for nearly every quantum application. Quantum-noise spectroscopy has been used in various quantum systems, such as superconducting qubits, nitrogen-vacancy centers, and trapped ions. Traditional spectroscopy methods are usually efficient in measuring noise spectra with mostly monotonically decaying contributions. However, there are important scenarios in which the noise spectrum is broadband and nonmonotonous, thus posing a challenge to existing noise-spectroscopy schemes. Here we compare several methods for noise spectroscopy: spectral decomposition based on the Carr-Purcell-Meiboom-Gill sequence, the recently presented dynamic sensitivity control (DYSCO) sequence, and a modified DYSCO sequence with a Gaussian envelope ( $\mathrm{gDYSCO}$ ). The performance of the sequences is quantified by analytic and numeric determination of the frequency resolution, bandwidth, and sensitivity, revealing a supremacy of gDYSCO to reconstruct nontrivial features. Using an ensemble of nitrogen-vacancy centers in diamond coupled to a high-density ${ }^{13} \mathrm{C}$-nuclear-spin environment, we experimentally confirm our findings. The combination of the schemes presented offers potential to record high-quality noise spectra as a prerequisite to generate quantum systems unlimited by their spin-bath environment.
\end{abstract}

DOI: 10.1103/PhysRevApplied.11.014064

\section{INTRODUCTION}

Quantum systems are inherently subject to noise originating from their coupling to the environment, which in turn affects their coherence properties, with implications for quantum-information processing, many-body dynamics, and quantum sensing [1-6]. As a consequence, studying the noise sources affecting quantum systems and optimizing schemes for mitigating them has been of great interest over the past decade [3-11]. Moreover, devising new quantum-noise spectroscopy methods (general techniques for analyzing noise sources using quantum probes) is a fundamental aspect of the field and has attracted significant attention over the past few years [9-13].

Quantum-noise spectroscopy has advanced in recent years, and has been used in the context of various quantum systems, such as superconducting qubits [10], trapped ions [14], ${ }^{13} \mathrm{C}$ atoms in adamantane [15], optically trapped ultracold atoms [16], and nitrogen-vacancy $(\mathrm{N}-V)$ centers in diamond $[11,12]$. These studies have led to a deeper

*yoav.romach@mail.huji.ac.il

†These authors contributed equally to this work. understanding of the physical origins and dynamics of the noise sources and of the system-environment interaction (see, e.g., Refs. [10-12]), as well as to advanced sensing applications (see, e.g., Ref. [14]).

In most of the previous studies, the spectrum of the relevant environmental noise was nearly monotonically decreasing (e.g., $1 / f$ noise [10], dc-centered Lorentzian $[11,12]$ ), bandwidth-limiting techniques were used [12], or the noise spectroscopy was performed with straightforward continuous driving, resulting, again, in limited bandwidth (see e.g., Ref. [16]). These approaches are therefore limited in addressing important situations commonly encountered in realistic systems, in which the contributing noise is distributed over a large frequency bandwidth but the noise spectrum also exhibits strong resonant features (and thus a nonmonotonous spectrum).

Here we investigate the potential of noise spectroscopy based on three microwave driving sequences: the CarrPurcell-Meiboom-Gill (CPMG) sequence postprocessed by spectral decomposition (CPMG SD) $[11,12]$ and the two recently introduced sequences dynamic sensitivity control (DYSCO) and a Gaussian enveloped DYSCO (gDYSCO) [17]. The properties of the sequences are 
studied analytically and numerically in terms of accessible bandwidth, frequency resolution, and gain as well as their implications for the reconstruction of noise spectra. Experiments are conducted with an ensemble of N- $V$ centers in diamond coupled to a bath of ${ }^{13} \mathrm{C}$ nuclear spins to confirm the predicted behavior.

The $\mathrm{N}-V$ center is a defect in the diamond lattice, in which one of the carbon atoms is replaced by a nitrogen atom and an adjacent site is replaced by a vacancy. A zero-field splitting of approximate $2.87 \mathrm{GHz}$ between $m_{s}=0$ and $m_{s}= \pm 1$ defines the triplet ground state. By applying a static magnetic field, one can break the degeneracy between the $m_{s}= \pm 1$ substates through the Zeeman effect, thus creating an effective two-level system. The $\mathrm{N}-V$-center electron spin can be initialized and detected optically due to spin-dependent transitions, and can be coherently manipulated within the ground-state spin manifold with use of microwave fields [18].

In the last decade, the $\mathrm{N}-V$ color center in diamond has emerged as a leading platform for quantum information, quantum meteorology, and magnetic sensing [1,19-25] due to its remarkable properties, such as long coherence times at room temperature [18,26]. Methods adopted from the field of nuclear magnetic resonance, such as dynamical decoupling [27-30], increased the coherence time even further [26]. Substantial research efforts are being invested to understand the relevant noise sources affecting $\mathrm{N}-V$ centers and their physical origins, as well as to optimize protocols to suppress their adverse effects $[11,15,31]$.

Recently, N- $V$ centers have been used to measure submillihertz nuclear-magnetic-resonance (NMR) spectra [32-34]. In these studies, the authors took advantage of the long coherence times of nuclear spins, and/or implemented a locked-in or quantum-homodyne method to achieve enhanced spectral resolution. Glenn et al. [34] also applied a driving pulse on the nuclear spins to further increase the resolution. However, these measurements result in a relatively low spectral bandwidth, which is insufficient for a general noise-spectroscopy method aiming to reconstruct the entire noise spectrum, as is the focus here.

The system's decoherence due to environmentally induced noise can be expressed as $C(t)=e^{-\chi(t)}$, where $\chi(t)$ contains the dependence of the decoherence processes on the spectral noise density $S(\omega)$ through [35]

$$
\chi(t)=\int_{0}^{\infty} \frac{d \omega}{\pi} S(\omega) \frac{\tilde{F}(\omega t)}{\omega^{2}},
$$

where $\tilde{F}(\omega t)$ is the sequence-dependent frequency filter function (FF) defined as the absolute square of the Fourier transform of the time-dependent sequence sensitivity function. For convenience, we define $F(\omega t)=2 \tilde{F}(\omega t) / t \pi \omega^{2}$ such that $\int_{0}^{\infty} F(\omega t) d \omega=1$ (for a CPMG experiment).
Equation (1) becomes

$$
\chi(t)=\frac{t}{2} \int_{0}^{\infty} d \omega S(\omega) F(\omega t) .
$$

Generally, the system's sensitivity can be modulated in a pulsed or continuous manner. While pulsed sequences (e.g., CPMG ) flip the sensitivity between +1 and -1 , the continuously driven DYSCO scheme enables arbitrary modulation of the sensitivity. Precise driving of the quantum sensor enables tuning of the $F$ so as to measure the noise environment at well-defined sensing frequencies $f_{0}$. The full noise spectrum $S(\omega)$ can be reconstructed from a set of measurements [35].

\section{FILTER-FUNCTION ANALYSIS}

One of the simplest pulsed sensitivity-modulation sequences is the Hahn-echo sequence [36], in which a single $\pi$ pulse is applied in the middle of the sequence of length $t_{\text {Hahn }}=2 \tau_{\text {free }}$ to refocus the $\mathrm{N}-V$ spin and eliminate dc effects. Additionally, any noise contribution whose correlation time is shorter than the free evolution time $\tau_{\text {free }}$ cancels out. As an extension of the Hahn-echo experiment, the CPMG- $N$ pulse sequence [37] consists of $N$ equally spaced $\pi$ pulses with $2 \tau_{\text {free }}$ time intervals between them (Fig. 1). By changing the free precession time $\tau_{\text {free }}$, the experiment time $t_{\mathrm{CPMG}}$, and the number of pulses $N$, one can adjust the sensing frequency according to

$$
f_{0}^{\mathrm{CPMG}}\left(\tau_{\text {free }}\right) \approx \frac{N}{2 t_{\mathrm{CPMG}}}=\frac{1}{4 \tau_{\text {free }}} .
$$

The total experiment time is given by $t_{\mathrm{CPMG}}=2 N \tau_{\text {free }}$ and is ultimately limited by $2 T_{1}$ [38]. However, the pulsed nature of the sequence [i.e., stepwise sensitivity modulation; Fig. 2(a)] introduces higher harmonics into the filter function [Fig. 2(b)]. A significant contribution to the decoherence can originate from these higher harmonics if the

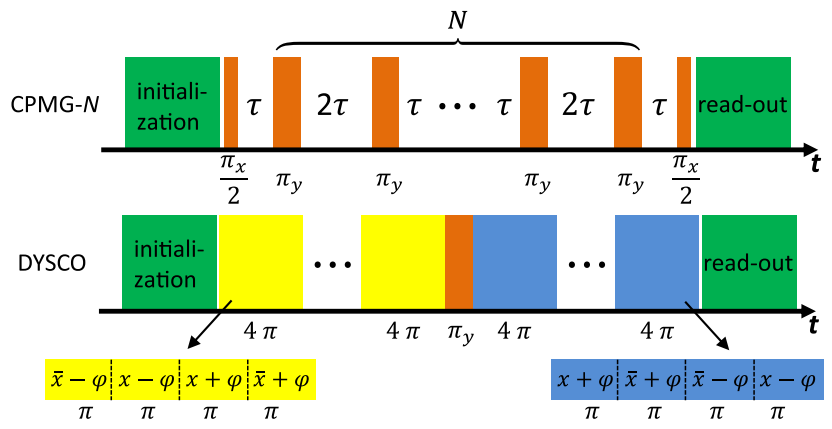

FIG. 1. The schematic for the CPMG and DYSCO experiments: green indicates a laser pulse, orange indicates a $\pi$ or $\pi / 2$ pulse, while yellow and blue indicate microwave-pulse blocks composed of $4 \pi$ pulses with the denoted phases (in which $\varphi$ is a function of the block number). 
noise spectrum is not monotonically decreasing. While the spectral decomposition scheme incorporates precise knowledge of the FF, the reconstructed spectrum nevertheless suffers from artifacts of this origin. (See the Appendix for a complete description of the spectral decomposition method).

The DYSCO pulse sequence was first presented by Lazariev et al. [17] as a means for selective radiofrequency spectroscopy using $\mathrm{N}-V$ centers. In contrast to spin-flipping sequences, the DYSCO sequence allows control of the instantaneous sensitivity of the spin sensor by precise pulse phase handling. This is achieved at the cost of permanent driving and reduction of the maximum sensitivity by a factor of $2 / \pi$. Modulation of the sensitivity function in a sinusoidal manner [Fig. 2(a)] results in a filter function free of higher harmonics. Given the finite experiment time, the DYSCO filter function is a squared sinc function that has small side lobes [Fig. 2(b)]. This effect can be suppressed by the addition of a Gaussian envelope to the sensitivity modulation (gDYSCO) [Fig. 2(a)], which removes the side lobes at the cost of further reduced sensitivity and a slightly increased width of the main peak [Fig. 2(b)].

For a quantitative analysis of the FFs, we examine four parameters: resolution, minimum frequency $f_{\min }$, maximum frequency $f_{\max }$, and the gain $\Sigma$ (a measure for the system's response at the desired frequency). While $f_{\min }$ and $f_{\max }$ define the system's bandwidth, we introduce the FWHM of the FF main peak as a quantitative measure of the frequency resolution. However, it should be stressed that the FF side lobes (for CPMG and DYSCO experiments) limit the resolution by increasing the effective envelope around the main peak. Therefore, the FWHM gives only a lower bound on the resolution.
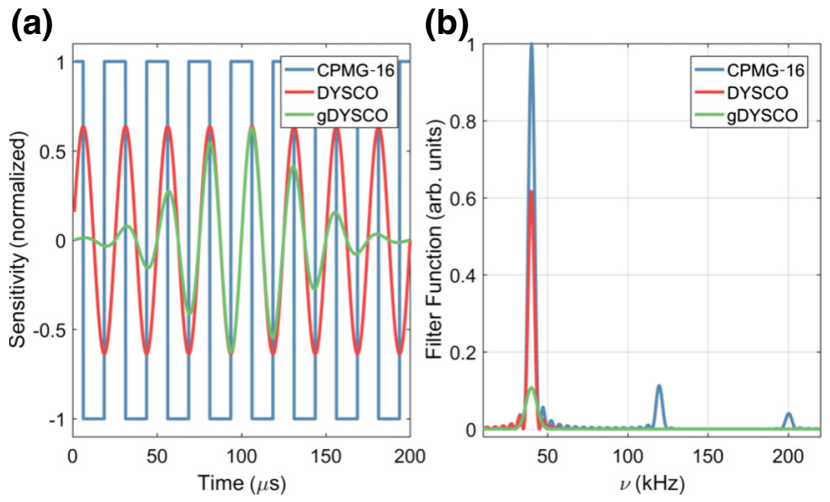

FIG. 2. The time-domain sensitivity functions for CPMG-16, DYSCO, and gDYSCO experiments (a) and the respective FFs (b). The higher harmonics in the CPMG FF do not appear for DYSCO FF at the cost of a smaller maximum filter-function height. When a Gaussian envelope is added, the side lobes disappear at the cost of an even further reduced maximum filter-function height and an increased width of the main peak.
The DYSCO FF is a squared sinc function such that the FWHM is approximately $0.884 / t_{\mathrm{DYSCO}}$ (calculated numerically), where $t_{\mathrm{DYSCO}}$ is the experiment time, ultimately limited by $T_{1 \rho}$ (the relaxation time in the driven system). On the other hand, the gDYSCO FF is a Gaussian with FWHM of $\sqrt{\ln 2} / \pi \sigma \approx 0.265 / \sigma$, where $\sigma$ is the width of the time-domain Gaussian envelope; $\sigma$ is limited by the experiment time, and in our experiments and simulation it is chosen to be $t_{\mathrm{DYSCO}} / 6$ such that the envelope approaches 0 at the beginning and end of the sequence. This results in a gDYSCO FWHM of approximately $1.59 / t_{\mathrm{DYSCO}}$. In contrast, the CPMG FF main peak frequency and FWHM can be derived only numerically. The FWHM is approximately $0.89 / t_{\mathrm{CPMG}}$. It is important to note that there is a significant difference between the CPMG experiment time, $t_{\mathrm{CPMG}}$, and the DYSCO experiment time, $t_{\mathrm{DYSCO}}$. In a DYSCO experiment, $t_{\mathrm{DYSCO}}$ remains constant regardless of the sensing frequency. However, in a CPMG experiment, $t_{\mathrm{CPMG}}$ changes with the sensing frequency, which is given in Eq. (3). It follows that the CPMG FF FWHM can also be expressed as being approximately $1.77\left(f_{0} / N\right)$ or approximately $0.89 / 2 \tau_{\text {free }} N$. In addition, $t_{\mathrm{DYSCO}}$ is limited by $T_{1 \rho}$ due to its continuously driven nature, while $t_{\mathrm{CPMG}}$ is limited by $T_{2}$ (the transverse, spin-spin, relaxation time), which depends on $N$ and is ultimately limited by $T_{1}$ (the longitudinal, spin-lattice, relaxation time). These nontrivial relations complicate the comparison between DYSCO and CPMG experiments. However, it can be generally stated that a CPMG experiment will achieve a higher resolution (narrower FF) around a given frequency by using a high number of pulses.

The gain $\Sigma$ is defined by the integral over the main peak of the FF in the region of the FWHM:

$$
\Sigma=\int_{f_{0}-\Delta}^{f_{0}+\Delta} F(f) d f,
$$

where $f_{0}$ is the FF peak frequency and $2 \Delta$ is the FWHM. The gain, $\Sigma$, is a measure of how much the coherence curve is affected by the presence of noise around the sensed frequency and is only weakly dependent on $f_{0}$. The DYSCO gain $\Sigma_{\text {DYSCO }}$ is approximately $60 \%$ of the CPMG gain $\Sigma_{\text {CPMG }}$ [17] and the gDYSCO gain $\Sigma_{\text {gDYSCO }}$ is approximately $20 \%$ of the $\Sigma_{\mathrm{CPMG}}$.

The maximum frequency for a CPMG experiment is limited by the requirement $2 \tau_{\text {free }} \gg \tau_{\pi}$, where $\tau_{\pi}$ is the duration of a $\pi$ pulse. As a consequence, the maximum frequency is given by $f_{\max }^{\mathrm{CPMG}} \ll f_{\text {Rabi }}$ (with $f_{\text {Rabi }}$ being the Rabi, or the driving, frequency). On the other hand, in a DYSCO experiment, as well as in gDYSCO, the maximum frequency is limited by the quantization of the sensitivity sine function (due to discrete phase steps). Quantization of the sine to $n$ steps, where $n \gg 1$, leads to $n 4 \tau_{\pi} f_{\max }^{\text {DYSCO }} \ll 1 \Rightarrow f_{\max }^{\text {DYSCO }} \ll f_{\text {Rabi }} / 2 n$. Even though the expressions for $f_{\max }$ are similar, it needs to be stated 
that for small $n$ the DYSCO scheme sensitivity modulation approaches the steplike case of the CPMG scheme. The reduced DYSCO gain, $\Sigma_{\text {DYSCO}}$, in combination with the appearance of higher harmonics for small $n$, reveals the supremacy of the CPMG SD method to reconstruct the noise at high frequencies. The minimum frequency for a CPMG experiment is estimated in a similar manner and is $f_{\min }^{\text {CPMG }} \lesssim 1 / 2 T_{2}^{\text {echo }}$ (see the Appendix for the derivation). For DYSCO and gDYSCO experiments the minimum frequency is given by the demand to fit at least one full period of the sensed frequency in the total experiment time, so $f_{\text {min }}^{\text {DYSCO }} t_{\mathrm{DYSCO}}>1 \Rightarrow f_{\text {min }}^{\text {DYSCO }}>1 / t_{\mathrm{DYSCO}}$. Recall that the timescale in DYSCO is limited by $T_{1 \rho}$. Therefore, CPMG has a clear advantage in terms of bandwidth, or dynamic range.

\section{SIMULATIONS}

Numerical simulations demonstrate the limitations of the different methods. As a proof of principle, the trivial case of a monotonically decreasing noise spectrum $S(\omega)$ is considered. By integrating Eq. (2) for a set of CPMG FFs (see the Appendix for more information), we simulate complete coherence curves. Addition of normally distributed noise to the coherence curves generates realistic data sets with experimental uncertainties [inset in Fig. 3(a)], which are subsequently analyzed by the CPMG SD method to reconstruct the spectral noise density. The results plotted in Fig. 3(a) show that for a monotonically decreasing noise spectrum the CPMG SD method reconstructs the noise spectrum with high precision. Repeating the procedure for the DYSCO FF reveals the noise spectrum with similar accuracy. In the next step a Gaussian peak around $62 \mathrm{kHz}$ is added to the initial noise spectrum, which simulates a ${ }^{13} \mathrm{C}$ Larmor peak originating from surrounding nuclear spins at an external magnetic field of $|B| \approx 60$ G. Figures 3(b)-3(d) depict the reconstruction of the simulated noise spectra with the CPMG SD, the DYSCO scheme and the gDYSCO scheme, respectively. The spectral curves show the reconstruction with (red) and without (green) the addition of experimental uncertainty to the simulated coherence curves. The gDYSCO scheme results in a superior reconstruction of the ${ }^{13} \mathrm{C}$ peak, while CPMG SD suffers from a significant systematic bias caused by the higher harmonics and side lobes of the FF. When statistical noise is added, the limited sensitivity of the gDYSCO scheme alters the results, but the peak is still clearly visible as most of the noise affects the monotonous "background." In the CPMG SD case, the noise has a limited effect on this "background" but significantly flattens the noise peak. Additionally, it can be seen that the CPMG SD method, in the presence of noise, pushes the peak to slightly higher frequencies. This is caused by the asymmetry of the filter function and the higher harmonics.

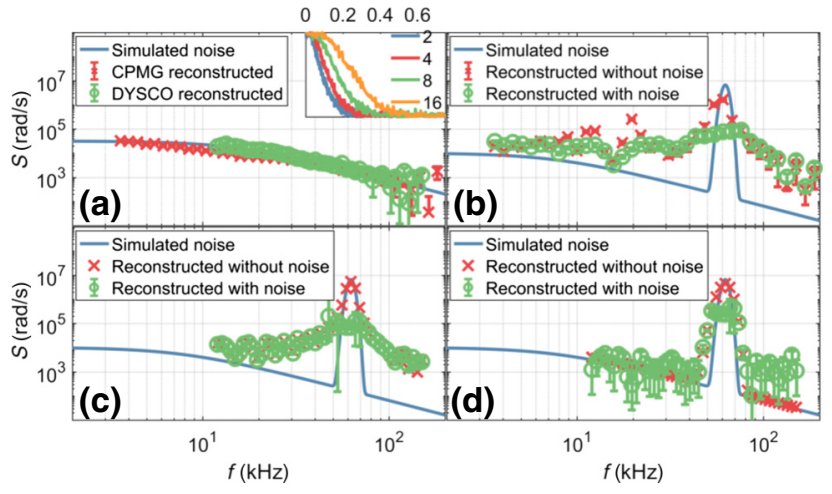

FIG. 3. Simulations of the various reconstructions methods. (a) A noise spectrum is defined as a dc-centered Lorentzian (blue). On the basis of the noise spectrum, CPMG- $N$ decoherence curves are generated (in the inset the numbers correspond to $N$, the $X$ axis is time in milliseconds, and the $Y$ axis is coherence in arbitrary units). Normally distributed noise added to the coherence curves simulates experimental uncertainties. Application of the decomposition code to the coherence curves reconstructs the noise spectrum (red). Equivalently, the reconstruction of the noise spectrum for the DYSCO sequence is simulated (green). (b)-(d) Extension of the analysis to noise spectra featuring a pronounced peak for CPMG- $N$, DYSCO, and gDYSCO schemes, respectively. The spectra obtained from a noise-free coherence curve are represented by the red data points, whereas the data represented by the green points incorporate the simulated experimental uncertainty. In general, the consideration of experimental uncertainties blurs the noise peak. Higher harmonics of the CPMG- $N$ FF cause artifacts in the reconstructed spectrum at roughly 30 and $15 \mathrm{kHz}$, which do not appear in the DYSCO and gDYSCO measurements. The gDYSCO scheme, which avoids the side lobes in the FF, reconstructs the noise spectrum with highest precision.

The simulated noise spectrum reconstructed with the gDYSCO scheme reproduces the noise peak with much higher precision than that reconstructed with the DYSCO scheme. Although the main peak of the DYSCO FF is a factor of 2 narrower than that of the gDYSCO FF, it still produces a much wider ${ }^{13} \mathrm{C}$ Larmor peak that is comparable to the one reconstructed by the CPMG SD method. This indicates a significant contribution originating from the side lobes of the DYSCO FF and highlights the fact that the resolution is not limited by the simple FF FWHM in the case of nonmonotonic spectra. Nevertheless, the FWHM gives a bound on the resolution, which is reached when the spectrum does not have a significant peak. Since both the CPMG FF and the DYSCO FF have feature-comparable side lobes, the resulting spectrum around the ${ }^{13} \mathrm{C}$ Larmor peak appears similar for both of them, with the CPMG FF higher harmonics contributing as a second-order effect.

\section{EXPERIMENT}

Experimental confirmation of our findings is obtained by the use of an ensemble of $\mathrm{N}-V$ centers coupled to a 


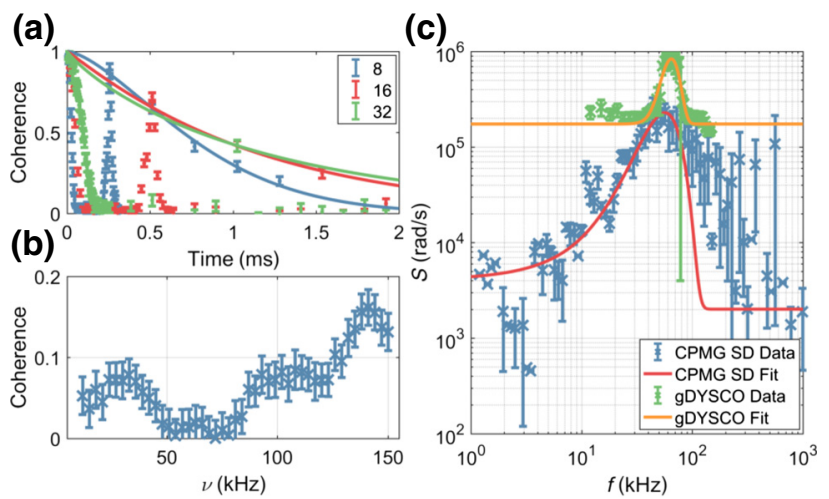

FIG. 4. (a) Experimental coherence curves for CPMG- $N$ experiments. Short timescales are densely sampled to reveal the ${ }^{13} \mathrm{C}$ collapse. The revival points are also sampled to extract the decoherence curve. The envelope functions $\exp \left[-(x / T)^{p}\right]$ fitted to the revival points are shown as solid lines. (b) Coherence curve obtained from a gDYSCO experiment as a function of the modulation frequency. (c) The reconstructed spectrum obtained with CPMG- $N$ experiments, with $N$ ranging from 2 to 128 , and the spectrum obtained from the gDYSCO experiment. Both spectra are fitted with a Gaussian; the fitted parameters are given in Table I. The ${ }^{13} \mathrm{C}$ Larmor peak reconstructed by gDYSCO is narrower than the one obtained by CPMG SD, as predicted from the simulation results [Fig. 3(b)]. In addition, it can be clearly seen that the limited sensitivity of gDYSCO does not reveal the noise spectrum when its power is below approximately $10^{5}(\mathrm{rad} / \mathrm{s})$ but results in a higher maximum detected noise spectrum (see the Appendix for more information).

bath of ${ }^{13} \mathrm{C}$ nuclear spins in a "off-the-shelf" diamond sample. The sample is a polished CVD-grown single-crystal diamond sample (Element Six, catalog no. 145-500-0248) featuring a natural ${ }^{13} \mathrm{C}$ abundance, a nitrogen concentration of less than $1 \mathrm{ppm}$, and a natural dense $\mathrm{N}-V$ ensemble with a concentration of approximately $1 \mathrm{ppb}$. We perform CPMG experiments on two timesscales. Short-timescale measurements with high temporal resolution allow us to extract the coherence revival caused by the Larmor precession, and thereby the magnetic field. In contrast, long-timescale measurements sampling only the Larmor revivals extract the coherence-curve envelope. These two experiment classes allow us to reconstruct both the ${ }^{13} \mathrm{C}$ Larmor peak and the long-time, low-frequency component of the noise. Additionally, DYSCO and gDYSCO experiments are performed, each with a total experiment time of $t_{\mathrm{DYSCO}}=200 \mu \mathrm{s}$. The experiments are performed by applying the pulse sequences shown in Fig. 1. The raw experimental data for the CPMG and gDYSCO experiments are presented in Fig. 4(a) and 4(b) and the resulting (reconstructed) spectra are presented in Fig. 4(c) (data for the normal DYSCO experiment can be found in the Appendix). To extract the central frequency and width, the ${ }^{13} \mathrm{C}$ Larmor peak is fitted by a Gaussian function (Fig. 4) (exact values are given in Table I). For comparison, on the
TABLE I. ${ }^{13} \mathrm{C}$ Larmor frequency as extracted from the reconstructed experimental noise spectra by means of a Gaussian fit. The width of the Larmor feature is the fitted Gaussian $1 \sigma$.

\begin{tabular}{lcc}
\hline \hline & CPMG & gDYSCO \\
\hline$f(\mathrm{kHz})$ & $60.2 \pm 4.2$ & $64.3 \pm 0.5$ \\
Width $(\mathrm{kHz})$ & $29.1 \pm 2.2$ & $13.8 \pm 0.8$ \\
\hline \hline
\end{tabular}

basis of the external magnetic field, the Larmor frequency is determined to be $f_{\text {Larmor }}=62.5(6) \mathrm{kHz}$.

Figure 4(c) highlights the benefits of the different methods. Large-bandwidth information is recovered by the CPMG SD, spanning a frequency range 3 orders of magnitude larger than for the gDYSCO scheme. Within this range, the sensitivity dynamic ratio is higher by at least 2 orders of magnitude, thereby giving a significantly improved estimate on the upper bound of the noise contribution. However, the ability to resolve narrow resonant features within the spectrum is significantly improved for the gDYSCO scheme.

\section{DISCUSSION}

We briefly compare our work with three recent studies that we became aware of during the preparation of the manuscript [39-41]. In Ref. [39] adaptive XY- $n$ (AXY- $n$ ) sequences are introduced, which are non-equally-spaced $\mathrm{XY}-n$ sequences. The AXY- $n$ sequence also suppresses the higher harmonics present in the CPMG sequence and could, theoretically, be used for noise spectroscopy. However, there are two caveats for using a AXY- $n$ scheme compared with the gDYSCO scheme: (i) because of the finite sequence duration, the AXY- $n$ FF will still have the same side lobes as the CPMG and DYSCO FFs, which will result in decreased spectral resolution; (ii) the AXY- $n$ FF will still have other spurious peaks, which might have to be decomposed in a similar SD manner. However, it is possible that the location of these spurious peaks can be controlled well enough for this to be ignored. In addition, the DYSCO scheme is composed of continuous driving with frequent phase changes, which increases its susceptibility to pulse errors. Overall, it seems that an AXY SD might have an advantage compared with CPMG SD, but an in-depth comparison encompassing also the gDYSCO scheme merits further study. In Refs. [40,41], the authors suggest the use of discrete prolate spheroidal sequences (DPSSs) [42] to probe and identify fine spectral features (resonant peaks). The DPSSs FF, like the DYSCO and gDYSCO FF, do not have the higher harmonics (sometimes referred to in the literature as "spectral leakage" or "Gibbs artifacts") present in the CPMG FF. In addition, they have reduced side lobes, less than the CPMG and DYSCO FFs, but more than the gDYSCO FF. In these studies, the authors used simulations to characterize the noise spectroscopy using DPSSs. Therefore, a direct comparison with the gDYSCO 
scheme in terms of sensitivity, bandwidth, and resolution is nontrivial and is beyond the scope of the current work.

In conclusion, we analyze and compare different quantum-noise-spectroscopy methods for the case of nonmonotonic spectra, specifically containing a large and narrow resonant feature. We find that the gDYSCO scheme provides higher resolution and allows more-precise detection of distinct features, while spectral decomposition based on CPMG exhibits higher sensitivity and larger bandwidth. Therefore, this work suggests that the best approach is a combination of different approaches so as to reveal full spectral information of nontrivial noise baths (our results indicate that optimized conditions might increase the sensitivity of gDYSCO, as described in the Appendix). This insight could provide an important tool for the study and characterization of a wide range of quantum systems, such as various solid-state defects, superconducting circuits, quantum dots, and trapped ions, leading to a deeper understanding of the relevant physical processes, as well as optimized control schemes for quantum applications such as sensing and quantum-information processing. In particular, this method could be used in an NMR or MRI (magnetic-resonance-imaging) measurements, traditionally done with pulsed XY-8 sequences, to increase the sensitivity, bandwidth, and accuracy. Other studies that have found nonmonotonous magnetic noise, such as pulsed-modulation measurements done with ions [14], could also benefit from these new methods.

\section{ACKNOWLEDGMENTS}

We thank Stefan Hell for the use of laboratory equipment and for his support in the project. This work was supported in part by the Niedersachsen-Israel Cooperation Program (Volkswagen Stiftung), a Minerva ARCHES Award, the European Union (ERC StG), the CIFAR Azrieli Global Scholars program, the Ministry of Science and Technology, Israel, and the Israel Science Foundation (Grant No. 750/14). Y.R. is grateful for support from a Kaye Einstein Scholarship and from a CAMBR fellowship.

\section{APPENDIX}

\section{Spectral decomposition method for CPMG}

The CPMG- $N$ FF is given by the following expressions [35]:

$$
\begin{array}{ll}
\frac{16}{\pi t \omega^{2}} \sin ^{4}\left(\frac{\omega t}{4 N}\right) \sin ^{2}\left(\frac{\omega t}{2}\right) / \cos ^{2}\left(\frac{\omega t}{2 N}\right) & \text { for even } N, \\
\frac{16}{\pi t \omega^{2}} \sin ^{4}\left(\frac{\omega t}{4 N}\right) \cos ^{2}\left(\frac{\omega t}{2}\right) / \cos ^{2}\left(\frac{\omega t}{2 N}\right) & \text { for odd } N .
\end{array}
$$

Numerical study of the functions shows that they have a maximum at $\omega_{0} \approx \pi N / t$, with $t$ being the experiment time. This approximation is good for large $N$; the following table shows the deviation as a function of $N$ :

\begin{tabular}{rc}
\hline \hline$N$ & $\omega_{0} /(\pi N / t)$ \\
\hline 1 & 1.48 \\
2 & 2.30 \\
3 & 3.21 \\
4 & 4.17 \\
8 & 8.09 \\
16 & 16.04 \\
\hline \hline
\end{tabular}

The FWHM of the peak is given by $\Delta \omega \approx 1.77 \omega_{0} / N$. The area beneath this peak contains approximately $75 \%$ of the total FF area, with about $8 \%$ more in the side lobes, $10 \%$ in the second harmonic, which is at $\omega_{1} \approx 3 \omega_{0}$, and the rest in the higher harmonics.

To perform the spectral decomposition analysis, the following prerequisites need to be fulfilled:

(a) There is some cutoff at higher frequencies, and we are probing close enough to it (or after it) with several data points. For these data points, all of the contributions to $\chi$ come from the main lobe.

(b) The shortest CPMG data point is taken before any decoherence process affected the N- $V$ so that the coherence is maximal. This is important for the rescaling of the coherence curve and therefore $\chi$ and $S$. Of course, a small deviation is not critical.

(c) The FF main-lobe width is small compared with the changes in the spectrum such that the spectrum is approximately linear inside the main lobe. This is much easier to achieve for high CPMG $N$.

(d) Alternatively, the spectrum is probed densely, such that adjacent data points have overlapping FFs and the spectrum does not change much between them.

The SD is done in two steps: the first step assumes that the main contribution comes from the first harmonic, which is treated as a rectangular function with the same width and area as the main-peak FWHM. An initial spectrum is calculated by approximating Eq. (2):

$$
\begin{aligned}
\chi(t) & \approx \frac{t}{2} \int_{\omega_{0}-\Delta}^{\omega_{0}+\Delta} S(\omega) F(\omega t) d \omega \\
& \approx \frac{t}{2} S\left(\omega_{0}\right) \int_{\omega_{0}-\Delta}^{\omega_{0}+\Delta} F(\omega t) d \omega \\
& \approx \frac{t}{2} S\left(\omega_{0}\right) \Sigma .
\end{aligned}
$$


This leads to the first-order approximation

$$
S_{0}\left(\omega_{0}\right)=-\frac{2 \chi(t)}{t \Sigma}
$$

where $\Sigma$, the gain, is the area under the main peak and is given by Eq. (4). The second step corrects for the higher harmonics by subtracting their contribution; the secondorder correction is

$$
S\left(\omega_{0}\right)=S_{0}\left(\omega_{0}\right)-\int_{\omega_{0}+\Delta}^{\infty} S(\omega) F(\omega t) d \omega .
$$

This equation is applied iteratively for all points, starting from the highest-frequency point downward. Because of the overlap in frequency points resulting from the different CPMG- $N$ curves, the resulting spectrum is very "dense." To extract a more-eye-friendly figure, the data are then binned, with the error bars corresponding to the within-bin spread.

\section{CPMG scheme minimum and maximum sensing frequency}

The main peak of the CPMG FF is at $f_{0} \approx N / 2 t=1 / 4 \tau$, where $2 \tau$ is the time between pulses in the CPMG experiment. For a pulsed experiment, we have to maintain the condition $2 \tau \gg \tau_{\pi}$, where $\tau_{\pi}=1 / 2 f_{\text {Rabi }}$ is the pulse duration and $f_{\text {Rabi }}$ is the Rabi frequency. From these relations we can extract $f_{\max } \ll f_{\text {Rabi }}$.

For the minimum frequency, we need to look again at $f_{0} \approx N / 2 t$. We need to maximize $t$ while minimizing $N$. In the presence of spin-bath noise, the coherence time for the CPMG experiment is given by $T_{2}(N)=T_{2}(1) N^{p}$, where $T_{2}(1)$ is the Hahn-echo time and $0<p<1$ [38]. These relations results in a sensing frequency which is given by $f_{0} \approx\left(1 / 2 T_{2}(1)\right) N^{1-p}$. From this, it is clear that the lowest frequency will be given in a Hahn-echo experiment $\left[T_{2}(1) \equiv T_{2}^{\text {echo }}\right]$. In practice, this frequency will be given by the longest time in a Hahn-echo experiment such that we still have a high-enough SNR, which is usually 2-4 times $T_{2}^{\text {echo }}$. This means that the lowest-possible sensing frequency for CPMG is $f_{\min } \lesssim 1 / 2 T_{2}^{\text {echo }}$.

\section{Hahn-echo and DYSCO coherence curves}

The lowest frequency that can be probed by the CPMG scheme is $f_{\min } \approx 1 / 2 T_{2}^{\text {echo }}$. We measured $T_{2}^{\text {echo }}=488 \pm$ $85 \mu \mathrm{s}$ [Fig. 5(a)] and therefore $f_{\min } \approx 1 \mathrm{kHz}$ follows, which is in good agreement with the data presented in Fig. 4. In contrast, the lowest measurable frequency in a DYSCO experiment is equivalent to the inverse of the experiment length, which is $200 \mu \mathrm{s}$. To find the optimal working point, we perform a DYSCO experiment with zero sensitivity at all times [Fig. 5(b)]. The DYSCO experiment length is chosen to be at the first revival point. Working at this point provides us with enough spectral resolution to perform the (a)

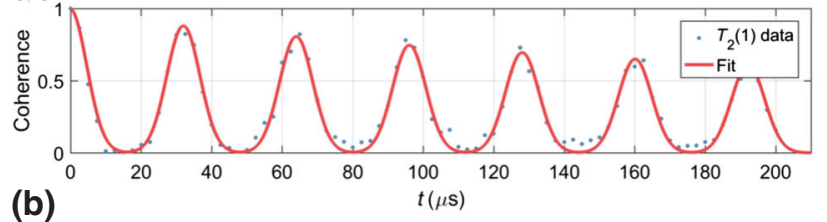

(b)

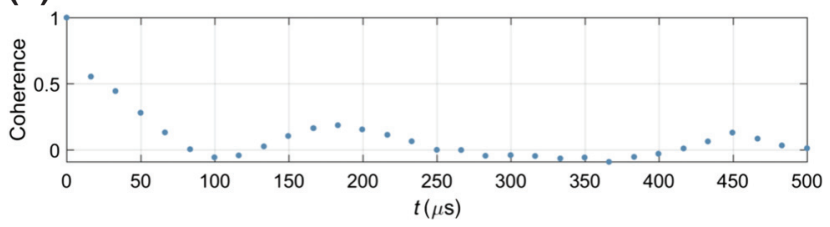

FIG. 5. (a) The coherence curve obtained for a Hahn-echo $\left(T_{2}(1)\right)$ experiment. The function is fitted to a sum of Gaussians centered around the Larmor revivals multiplied by an envelope $e^{-\left(t / T_{2}\right)^{p}}\left[\sum_{i} e^{-\left(t-i t_{t}\right)^{2}} / 2 \sigma^{2}\right]$, where $i$ ranges from 0 to 6 , and the decay time $T_{2}=488 \mu \mathrm{s}$. (b) The coherence curve obtained for a DYSCO experiment with zero sensitivity at all times as a function of the experiment time. The measurements reported in the main text are done at $200 \mu \mathrm{s}$, near the first peak in the curve. The occurrence of collapses and revivals is not well understood and will be the subject of further investigations.

experiments and to distinguish the ${ }^{13} \mathrm{C}$ peak. It is possible to work at the second revival point at $400 \mu \mathrm{s}$ if higher spectral resolution is needed. Under optimal conditions $t_{\mathrm{DYSCO}}$ approaches $T_{1 \rho}$ [17], which is measured to be 7.3(9) $\mu \mathrm{s}$. However, the unpolarized nuclear spin in combination with driving-field imperfections significantly reduces $t_{\mathrm{DYSCO}}$. A more-careful magnetic field alignment, which will significantly increase the nuclear spin polarization, will most likely increase $t_{\mathrm{DYSCO}}$ and move it toward $T_{1 \rho}$.

\section{Noise-spectrum simulation versus experiment}

The noise spectrum in the experimental configuration originates from two main sources: the ${ }^{14} \mathrm{~N}$ spin bath, which can be modeled as a dc-centered Lorentzian, and the ${ }^{13} \mathrm{C}$ spin bath, which can be modeled as a Gaussian around the Larmor frequency:

$$
\begin{aligned}
S(\omega)= & \frac{\Delta_{{ }_{13} \mathrm{C}}^{2}}{\sqrt{2 \pi} \sigma_{13} \mathrm{C}} \exp \left[-\frac{\left(\omega-\omega_{\text {Larmor }}\right)^{2}}{2 \sigma_{13}^{2} \mathrm{C}}\right] \\
& +\frac{\Delta_{14}^{2} \mathrm{~N}}{\pi \sigma_{14 \mathrm{~N}}\left[1+\left(\frac{\omega}{\sigma_{14}}\right)^{2}\right]},
\end{aligned}
$$

where the $\Delta$ are the respective coupling strengths, the $\sigma$ are the respective widths, and $\omega_{\text {Larmor }}$ is the ${ }^{13} \mathrm{C}$ Larmor frequency. To obtain a simulated noise spectrum with realistic values, the following procedure is used: A noise spectrum is generated, the CPMG- 8 coherence curve is created from the spectrum, and then the simulated CPMG- 8 curve is 


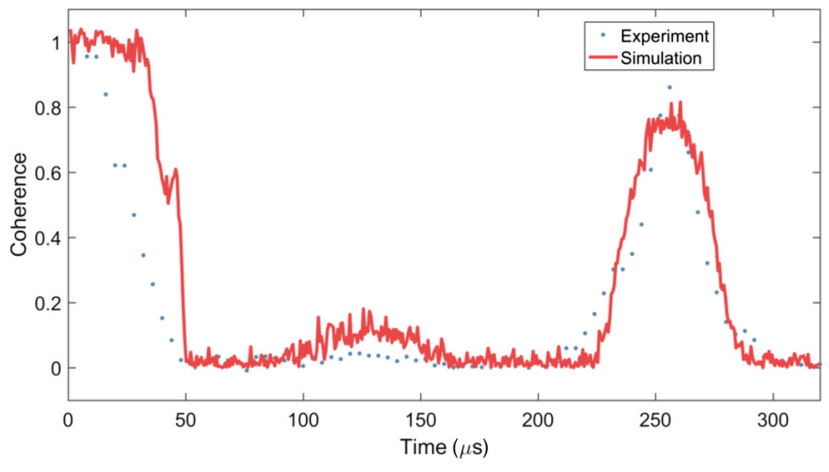

FIG. 6. The experimental results for CPMG-8 experiments (blue) and the simulated CPMG-8 data (red).

compared with the experimental one. A norm 2 minimization problem is then solved by changing the noise parameters to minimize the difference between the simulation and the experimental data. This results in the following parameters:

$$
\begin{aligned}
\Delta_{13 \mathrm{C}} & \approx 500 \mathrm{krad} / \mathrm{s}, \quad \sigma_{13 \mathrm{C}} \approx 25 \mathrm{krad} / \mathrm{s}, \\
\Delta_{{ }^{14} \mathrm{~N}} & \approx 40 \mathrm{krad} / \mathrm{s}, \quad \sigma_{14_{\mathrm{N}}} \approx 50 \mathrm{krad} / \mathrm{s}, \\
\omega_{\text {Larmor }} & \approx 392 \mathrm{krad} / \mathrm{s} .
\end{aligned}
$$

The comparison between the simulation and experiment can be found in Fig. 6. While the fine details are different, the main behavior is similar. The values obtained are used to generate the spectra shown in Fig. 3.

\section{Sensitivity and gain}

The sensitivity of the different schemes should take into account the different gains, $\Sigma$, and the measurement uncertainty $\epsilon$ (Fig. 7). The coherence is defined as $C(t)=e^{-\chi(t)}$, where $\chi(t)$ is given in Eq. (2). When the FF is approximated to the Dirac $\delta$ function, $\chi(t)$ is simplified as given in Eq. (A2). Substitution of this into the expression for the coherence gives

$$
\begin{aligned}
C(t) & =e^{\frac{t}{2} S\left(\omega_{0}\right) \Sigma}, \\
\ln C(t) & =-\Sigma \frac{t}{2} S\left(\omega_{0}\right), \\
S\left(\omega_{0}\right) & =-\frac{2 \ln C(t)}{t \Sigma},
\end{aligned}
$$

where $t$ is the experiment duration and $\Sigma$ (gain) is the area underneath the main peak of the FF. Assuming that the coherence measurement has uncertainty with a standard deviation of $\epsilon$, the coherence that we can detect for CPMG is $\epsilon \lesssim C(t) \lesssim 1-\epsilon$. If we substitute this into Eq. (A7), the

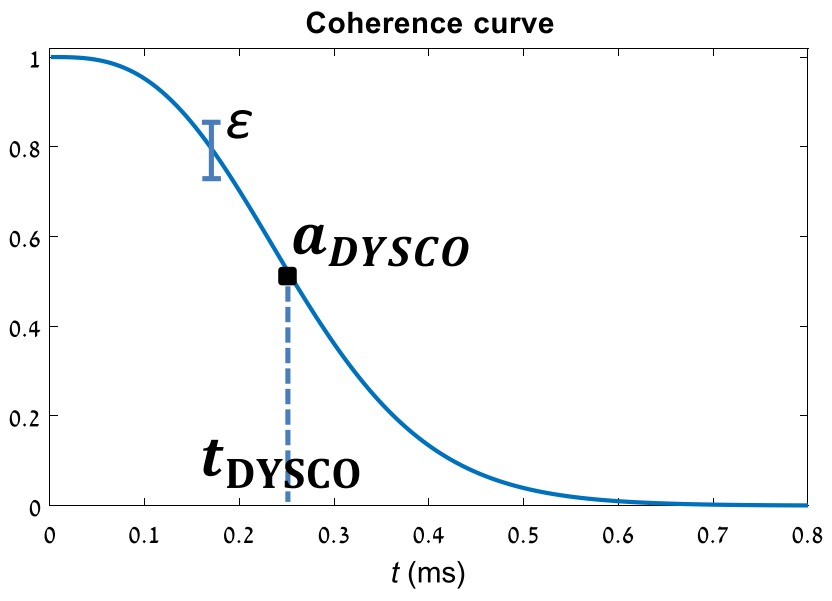

FIG. 7. Coherence decay curve. Superimposed are the relevant magnitude of DYSCO maximum contrast, $a_{\mathrm{DYSCO}}$, and uncertainty, $\epsilon$.

signal range will be

$$
S\left(\omega_{0}\right)=-\frac{2}{t \Sigma_{\mathrm{CPMG}}} \begin{cases}\ln (1-\epsilon), & S_{\min }, \\ \ln \epsilon, & S_{\max }\end{cases}
$$

For DYSCO, a specific time, $t_{\mathrm{DYSCO}}$, is chosen. At this time, the contrast, $a_{\mathrm{DYSCO}}$, is smaller than 1 and it is the maximum DYSCO-experiment contrast. Repeating the calculation for DYSCO, we obtain

$$
S\left(\omega_{0}\right)=-\frac{2}{t \Sigma_{\text {DYSCO }}} \begin{cases}\ln \left(a_{\text {DYSCO }}-\epsilon\right), & S_{\min }, \\ \ln \epsilon, & S_{\max } .\end{cases}
$$

See Fig. 7 for an illustration and Table II for the values of $\Sigma$.

If the measurement uncertainty is $3 \%$, it follows that CPMG can detect 2 orders of magnitude $S_{\max } / S_{\min } \approx 100$. If a DYSCO measurement is made such that the contrast is dropped to $80 \%\left(a_{\mathrm{DYSCO}}=0.8\right)$, then the ratio for DYSCO drops to $S_{\max } / S_{\min } \approx 10$. The lower gain of gDYSCO allows it to detect a higher-noise spectrum, compared with CPMG, higher by a factor of approximately 6 , in accordance with the experimental results in Fig. 4(c).

\section{FFT windows}

In the language of signal processing, gDYSCO is similar to a Gaussian window applied to a signal before a discrete

TABLE II. The gain, $\Sigma$, for the different schemes, calculated for the FWHM.

\begin{tabular}{lll}
\hline \hline Scheme & $\Sigma$ \\
\hline CMPG & 0.6 \\
DYSCO & 0.35 \\
gDYSCO & 0.11 \\
\hline \hline
\end{tabular}


Fourier transform is applied to it. In a similar manner, the DPSSs [40-42] are similar to a Slepian window [43]. There is extensive research into the windows that give the largest rms time-bandwidth product, with the Gaussian window giving an almost optimal value [44]. It is possible to use a better window, such as the cosine window [44], to further increase the resolution by a small amount.

\section{Confocal setup}

Experiments are performed with a confocal microscope capable of coherent spin manipulation. Excitation light is provided by a pulsed laser with 532-nm wavelength and a repetition rate of $30 \mathrm{MHz}$. An acousto-optical modulator (AOM) in a double-pass configuration allows fast on-off switching of the excitation light while guaranteeing an extinction ratio of approximately $66 \mathrm{~dB}$ in the off state. The laser is focused onto the diamond with use of a high-NA objective (Leica 1.47). The fluorescence signal in the wavelength window from 580 to $740 \mathrm{~nm}$ is collected through the same objective, then directed through a dichroic mirror and finally detected by means of an avalanche photodiode and stored in a multiple-event time digitizer. The AOM is controlled by an arbitrary-waveform generator (Tektronix), which at the same time is providing the microwave signal to the sample by a microwire antenna. The fluorescence signal read during the "readout" period (Fig. 1) is normalized by the counts at the end of the "initialization" period, which immediately follows. This results in normalization with respect to the excitation laser intensity, greatly reducing the noise caused by fluctuations in the laser intensity, which originate from the laser itself, the AOM, and from mechanical instabilities. Because of the high diamond refractive index, the collection efficiency is approximately $10 \%$ [45]. Combined with the coupling to the avalanche photodiode and its quantum efficiency, this results in a photon-detection efficiency of

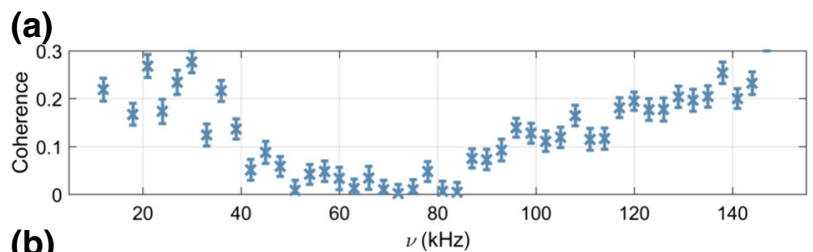

FIG. 8. (a) The coherence curve obtained from a DYSCO measurement as a function of the DYSCO modulation frequency. (b) The spectrum obtained from the DYSCO measurements with a fitted Gaussian centered around $67.1(2) \mathrm{kHz}$ with a width of $17.3(2) \mathrm{kHz}$. approximately $2 \%$ for the fluorescence signal. The limiting noise source in the setup is shot noise, which is amplified by the low photon-detection efficiency [45]. A single read-out duration is $300 \mathrm{~ns}$, with an average of one photon per read-out. Each experiment is performed $1 \times 10^{6}$ times, corresponding to a relative shot noise of about $0.1 \%$. This is to be compared against the contrast, which is on the order of $1 \%-3 \%$, giving a SNR on the order of $10-30$, similar to that in other studies [46].

\section{DYSCO results}

The results from the regular DYSCO are presented in Fig. 8. These results suffer both from the wide peak due to the side lobes and from the reduced sensitivity of DYSCO.

[1] T. Ladd, F. Jelezko, R. Laflamme, Y. Nakamura, C. Monroe, and J. O'Brien, Quantum computers, Nature 464, 45 (2010).

[2] Alexander Shnirman, Yuriy Makhlin, and Gerd Schn, Noise and decoherence in quantum two-level systems, Phys. Scr. 2002, 147 (2002).

[3] G. Ithier, E. Collin, P. Joyez, P. J. Meeson, D. Vion, D. Esteve, F. Chiarello, A. Shnirman, Y. Makhlin, J. Schriefl, and G. Schön, Decoherence in a superconducting quantum bit circuit, Phys. Rev. B 72, 134519 (2005).

[4] Paula I. Villar and Fernando C. Lombardo, Decoherence of a solid-state qubit by different noise correlation spectra, Phys. Lett. A 379, 246 (2015).

[5] John M. Martinis, K. B. Cooper, R. McDermott, Matthias Steffen, Markus Ansmann, K. D. Osborn, K. Cicak, Seongshik Oh, D. P. Pappas, R. W. Simmonds, and Clare C. Yu, Decoherence in Josephson Qubits from Dielectric Loss, Phys. Rev. Lett. 95, 210503 (2005).

[6] J. Wenner, R. Barends, R. C. Bialczak, Yu Chen, J. Kelly, Erik Lucero, Matteo Mariantoni, A. Megrant, P. J. J. O’Malley, D. Sank, A. Vainsencher, H. Wang, T. C. White, Y. Yin, J. Zhao, A. N. Cleland, and John M. Martinis, Surface loss simulations of superconducting coplanar waveguide resonators, Appl. Phys. Lett. 99, 113513 (2011).

[7] J. R. Klauder and P. W. Anderson, Spectral diffusion decay in spin resonance experiments, Phys. Rev. 125, 912 (1962).

[8] J. Medford, łL. Cywiński, C. Barthel, C. M. Marcus, M. P. Hanson, and A. C. Gossard, Scaling of Dynamical Decoupling for Spin Qubits, Phys. Rev. Lett. 108, 086802 (2012).

[9] Fei Yan, Simon Gustavsson, Jonas Bylander, Xiaoyue Jin, Fumiki Yoshihara, David G. Cory, Yasunobu Nakamura, Terry P. Orlando, and William D. Oliver, Rotating-frame relaxation as a noise spectrum analyser of a superconducting qubit undergoing driven evolution, Nat. Commun. 4, 2337 (2013).

[10] Jonas Bylander, Simon Gustavsson, Fei Yan, Fumiki Yoshihara, Khalil Harrabi, George Fitch, David G. Cory, Yasunobu Nakamura, Jaw-Shen Tsai, and William D. Oliver, Noise spectroscopy through dynamical decoupling with a superconducting flux qubit, Nat. Phys. 7, 565 (2011). 
[11] Y. Romach, C. Müller, T. Unden, L. J. Rogers, T. Isoda, K. M. Itoh, M. Markham, A. Stacey, J. Meijer, S. Pezzagna, B. Naydenov, L. P. McGuinness, N. Bar-Gill, and F. Jelezko, Spectroscopy of Surface-induced Noise using Shallow Spins in Diamond, Phys. Rev. Lett. 114, 017601 (2015).

[12] N. Bar-Gill, L. Pham, C. Belthangady, D. Le Sage, P. Cappellaro, J. Maze, M. Lukin, A. Yacoby, and R. Walsworth, Suppression of spin-bath dynamics for improved coherence of multi-spin-qubit systems, Nat. Commun. 3, 858 (2012).

[13] D. Farfurnik, N. Aharon, I. Cohen, Y. Hovav, A. Retzker, and N. Bar-Gill, Experimental realization of timedependent phase-modulated continuous dynamical decoupling, Phys. Rev. A 96, 013850 (2017).

[14] Shlomi Kotler, Nitzan Akerman, Yinnon Glickman, Anna Keselman, and Roee Ozeri, Single-ion quantum lock-in amplifier, Nature 473, 61 (2011).

[15] Gonzalo A. Alvarez and Dieter Suter, Measuring the Spectrum of Colored Noise by Dynamical Decoupling, Phys. Rev. Lett. 107, 230501 (2011).

[16] Ido Almog, Yoav Sagi, Goren Gordon, Guy Bensky, Gershon Kurizki, and Nir Davidson, Direct measurement of the system-environment coupling as a tool for understanding decoherence and dynamical decoupling, J. Phys. B: At. Mol. Opt. Phys. 44, 154006 (2011).

[17] Andrii Lazariev, Silvia Arroyo-Camejo, Ganesh Rahane, Vinaya Kumar Kavatamane, and Gopalakrishnan Balasubramanian, Dynamical sensitivity control of a single-spin quantum sensor, Sci. Rep. 7, 6586 (2017).

[18] Marcus W. Doherty, Neil B. Manson, Paul Delaney, Fedor Jelezko, Jörg Wrachtrup, and Lloyd C. L. Hollenberg, The nitrogen-vacancy colour centre in diamond, Phys. Rep. 528, 1 (2013).

[19] J. M. Taylor, P. Cappellaro, L. Childress, L. Jiang, D. Budker, P. R. Hemmer, A. Yacoby, R. Walsworth, and M. D. Lukin, High-sensitivity diamond magnetometer with nanoscale resolution, Nat. Phys. 4, 810 (2008).

[20] L. M. Pham, D. Le Sage, P. L. Stanwix, T. K. Yeung, D. Glenn, A. Trifonov, P. Cappellaro, P. R. Hemmer, M. D. Lukin, H. Park, A. Yacoby, and R. L. Walsworth, Magnetic field imaging with nitrogen-vacancy ensembles, New J. Phys. 13, 045021 (2011).

[21] F. Dolde, H. Fedder, M. Doherty, T. Nbauer, F. Rempp, G. Balasubramanian, T. Wolf, F. Reinhard, L. C. L. Hollenberg, F. Jelezko, and J. Wrachtrup, Electric-field sensing using single diamond spins, Nat. Phys. 7, 459 (2011).

[22] H. J. Mamin, M. Kim, M. H. Sherwood, C. T. Rettner, K. Ohno, D. D. Awschalom, and D. Rugar, Nanoscale nuclear magnetic resonance with a nitrogen-vacancy spin sensor, Science 339, 557 (2013).

[23] T. Staudacher, F. Shi, S. Pezzagna, J. Meijer, J. Du, C. A. Meriles, F. Reinhard, and J. Wrachtrup, Nuclear magnetic resonance spectroscopy on a (5-nanometer) ${ }^{3}$ sample volume, Science 339, 561 (2013).

[24] M. S. Grinolds, S. Hong, P. Maletinsky, L. Luan, M. D. Lukin, R. L. Walsworth, and A. Yacoby, Nanoscale magnetic imaging of a single electron spin under ambient conditions, Nat. Phys. 9, 215 (2013).

[25] L. Rondin, J.-P. Tetienne, S. Rohart, A. Thiaville, T. Hingant, P. Spinicelli, J.-F. Roch, and V. Jacques,
Stray-field imaging of magnetic vortices with a single diamond spin, Nat. Commun. 4, 2279 (2013).

[26] N. Bar-Gill, L. M. Pham, A. Jarmola, D. Budker, and R. L. Walsworth, Solid-state electronic spin coherence time approaching one second, Nat. Commun. 4, 1743 (2013).

[27] G. de Lange, Z. H. Wang, D. Ristè, V. V. Dobrovitski, and R. Hanson, Universal dynamical decoupling of a single solid-state spin from a spin bath, Science 330, 60 (2010).

[28] Boris Naydenov, Florian Dolde, Liam T. Hall, Chang Shin, Helmut Fedder, Lloyd C. L. Hollenberg, Fedor Jelezko, and Jörg Wrachtrup, Dynamical decoupling of a single-electron spin at room temperature, Phys. Rev. B 83, 081201 (2011).

[29] J. H. Shim, I. Niemeyer, J. Zhang, and D. Suter, Robust dynamical decoupling for arbitrary quantum states of a single NV center in diamond, Europhys. Lett. 99, 40004 (2012).

[30] C. A. Ryan, J. S. Hodges, and D. G. Cory, Robust Decoupling Techniques to Extend Quantum Coherence in Diamond, Phys. Rev. Lett. 105, 200402 (2010).

[31] M. Kim, H. J. Mamin, M. H. Sherwood, K. Ohno, D. D. Awschalom, and D. Rugar, Decoherence of Near-Surface Nitrogen-Vacancy Centers Due to Electric Field Noise, Phys. Rev. Lett. 115, 087602 (2015).

[32] Simon Schmitt, Tuvia Gefen, Felix M. Strner, Thomas Unden, Gerhard Wolff, Christoph Mller, Jochen Scheuer, Boris Naydenov, Matthew Markham, Sebastien Pezzagna, Jan Meijer, Ilai Schwarz, Martin Plenio, Alex Retzker, Liam P. McGuinness, and Fedor Jelezko, Submillihertz magnetic spectroscopy performed with a nanoscale quantum sensor, Science 356, 832 (2017).

[33] J. M. Boss, K. S. Cujia, J. Zopes, and C. L. Degen, Quantum sensing with arbitrary frequency resolution, Science 356, 837 (2017).

[34] David R. Glenn, Dominik B. Bucher, Junghyun Lee, Mikhail D. Lukin, Hongkun Park, and Ronald L. Walsworth, High-resolution magnetic resonance spectroscopy using a solid-state spin sensor, Nature 555, 351 (2018).

[35] łLukasz Cywinński, Roman M. Lutchyn, Cody P. Nave, and S. Das Sarma, How to enhance dephasing time in superconducting qubits, Phys. Rev. B 77, 174509 (2008).

[36] E. L. Hahn, Spin echoes, Phys. Rev. 80, 580 (1950).

[37] S. Meiboom and D. Gill, Modified spin-echo method for measuring nuclear relaxation times, Rev. Sci. Instrum. 29, 688 (1958).

[38] Rogerio de Sousa, in Electron Spin Resonance and Related Phenomena in Low-Dimensional Structures, edited by Marco Fanciulli, Series Topics in Applied Physics Vol. 115 (Springer, Berlin, Heidelberg, 2009), p. 183.

[39] J. Casanova, Z.-Y. Wang, J. F. Haase, and M. B. Plenio, Robust dynamical decoupling sequences for individualnuclear-spin addressing, Phys. Rev. A 92, 042304 (2015).

[40] V. M. Frey, S. Mavadia, L. M. Norris, W. Ferranti, D. Lucarelli, L. Viola, and M. J. Biercuk, Application of optimal band-limited control protocols to quantum noise sensing, Nat. Commun. 8, 2189 (2017).

[41] Leigh M. Norris, Dennis Lucarelli, Virginia M. Frey, Sandeep Mavadia, Michael J. Biercuk, and Lorenza Viola, Optimally band-limited spectroscopy of control noise using a qubit sensor, arXiv:1803.05538 [quant-ph] (2018). 
[42] D. Slepian, Prolate spheroidal wave functions, Fourier analysis, and uncertainty — V: The discrete case, Bell Syst. Tech. J. 57, 1371 (1978).

[43] F. J. Harris, On the use of windows for harmonic analysis with the discrete Fourier transform, Proc. IEEE 66, 51 (1978).

[44] Sebastian Starosielec and Daniel Hgele, Discrete-time windows with minimal RMS bandwidth for given RMS temporal width, Signal Process. 102, 240 (2014).
[45] D. Le Sage, L. M. Pham, N. Bar-Gill, C. Belthangady, M. D. Lukin, A. Yacoby, and R. L. Walsworth, Efficient photon detection from color centers in a diamond optical waveguide, Phys. Rev. B 85, 121202 (2012).

[46] D. Farfurnik, A. Jarmola, L. M. Pham, Z. H. Wang, V. V. Dobrovitski, R. L. Walsworth, D. Budker, and N. Bar-Gill, Optimizing a dynamical decoupling protocol for solid-state electronic spin ensembles in diamond, Phys. Rev. B 92, 060301 (2015). 